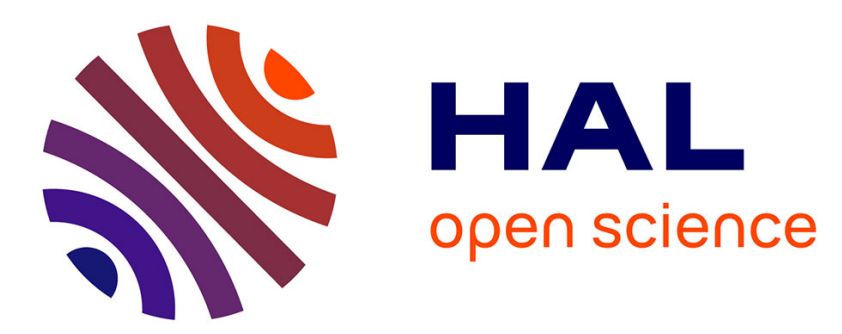

\title{
A la recherche d'une mutualisation des livraisons en milieu urbain: le cas du groupe NMPP
}

Jesus Gonzalez-Feliu, Joëlle Morana

\section{To cite this version:}

Jesus Gonzalez-Feliu, Joëlle Morana. A la recherche d'une mutualisation des livraisons en milieu urbain: le cas du groupe NMPP. Revue française de gestion industrielle, 2010, 29 (2), pp.71-92. halshs-00690097

\section{HAL Id: halshs-00690097 https://shs.hal.science/halshs-00690097}

Submitted on 21 Apr 2012

HAL is a multi-disciplinary open access archive for the deposit and dissemination of scientific research documents, whether they are published or not. The documents may come from teaching and research institutions in France or abroad, or from public or private research centers.
L'archive ouverte pluridisciplinaire HAL, est destinée au dépôt et à la diffusion de documents scientifiques de niveau recherche, publiés ou non, émanant des établissements d'enseignement et de recherche français ou étrangers, des laboratoires publics ou privés. 
Revue Française de Gestion Industrielle

Vol. $x x, n^{\circ}$ yy

\title{
A LA RECHERCHE D'UNE MUTUALISATION DES LIVRAISONS EN MILIEU URBAIN : LE CAS DU GROUPE NMPP $^{1}$
}

\author{
Jesús GONZALEZ-FELIU*, Joëlle MORANA*
}

Résumé. - La mutualisation des flux de transport est un sujet très populaire mais encore peu exploré. En milieu urbain, une mutualisation des flux de transport permet une meilleure rationalisation des moyens mis en place se traduisant à la fois par une réduction de coût et un impact environnemental moindre. Elle s'avère une alternative pour le secteur de la messagerie de presse, qui en France rencontre de plus en plus de difficultés à réduire ses coûts de distribution. Dans ce contexte, tout messager de presse, tel que le cas d'étude ici considéré, le groupe Nouvelles Messageries de la Presse Parisienne (NMPP), voit le taux de chargement de ses véhicules et entrepôts diminuer de manière constante. L'objet de ce travail est de faire un état des lieux des besoins, des marges de manœuvre et des choix envisageables pour obtenir un système performant de mutualisation des flux de transport, en l'appliquant au cas du groupe NMPP.

Mots clé: Logistique urbaine, mutualisation croisée, distribution de presse, systèmes d'information

\section{Introduction}

Le transport de marchandises est un élément important dans la chaîne d'approvisionnement et constitue un lien essentiel entre la demande et l'offre de marchandises. Ce secteur représente une importante source d'emplois, mais est confronté à des problèmes liés à la congestion et au respect de l'environnement, surtout en milieu urbain. De fait, avec le développement de l'espace urbain et de sa population, l'augmentation du nombre de voitures, les nuisances entraînées par une congestion de plus en plus forte, les émissions de $\mathrm{CO}_{2}$ et substances polluantes, le bruit et les questions sociales et sociétales (meilleure qualité de vie, etc.), les systèmes d'approvisionnements de la ville

\footnotetext{
${ }^{1}$ Laboratoire d'Economie des Transports, ISH, 14 Avenue Berthelot, 69363 Lyon Cedex 07

${ }^{1}$ Depuis le 14 décembre 2009, le groupe NMPP est devenu le groupe Presstalis.
} 
doivent être restructurés en commençant par les transports des marchandises en milieu urbain.

Depuis plus de quinze ans, la logistique urbaine est un sujet qui préoccupe autant les décideurs publiques (élus, techniciens des villes), les professionnels (logisticiens, opérateurs de transport) ou encore le monde de la recherche. Ce sujet ne cesse de faire l'objet de travaux de plus en plus nombreux et est au cœur de multiples colloques, échanges, initiatives, projets et expériences (Dablanc, 1998 ; Taniguchi et al., 2001 ; Patier, 2002 ; Routhier, 2002 ; Ambrosini and Routhier, 2004 ; Boudoin, 2006 ; Patier et al., 2007 ; Crainic, 2008 ; Taniguchi et Thomson, 2008 ; Delaître, 2009 ; Patier et Routhier, 2009a,b).

Afin de rationaliser les flux de marchandises, plusieurs solutions sont proposées. Les principales initiatives s'appuient sur la notion de Centre de Distribution Urbaine (CDU), où les flux de transport entrant dans la ville se concentrent pour remplir des véhicules légers, moins polluants et plus accessibles aux zones les plus denses et qui livrent les marchandises dans certaines zones de la ville principale. Cette stratégie, très populaire en début des années 2000, n'est efficiente que dans des cas particuliers, et a du mal à se reproduire sous un standard international : cf. des réussites ponctuelles en France (La Rochelle) et en Italie (Padoue et Parme principalement) ) et l'échec en Allemagne ou les Pays-Bas (Rosini, 2005). De plus, certaines alternatives ont eu des ennuis du point de vue juridique car elles s'appuyaient sur une réglementation très restrictive, à la limite du respect du libre marché. C'est le cas de VELOCE, le système de distribution de marchandises de la ville de Vicenza (Italie), où une réglementation excessivement restrictive oblige la grande majorité des catégories de marchandises à passer par le CDU (Spinedi, 2008). Une autre solution pour augmenter le taux de chargement est la mutualisation des flux de marchandises à l'aide d'une plate-forme virtuelle. Cette solution est moins développée, mais plusieurs études montrent son potentiel (Spinedi, 2008 ; Tremeac et Raux, 2009).

Dans la poursuite de ces réflexions, l'objectif de cet article est de réfléchir à la mise en place d'une stratégie de mutualisation des flux de transport, en s'appuyant sur l'idée de Systèmes d'Information et de Communication Logistique de l'Entreprise (SICLE : FabbeCostes, 1992, 1994, 1997, 2000 ; Livolsi et Fabbe-Costes, 2004). A l'instar de Seiersen (2006), il convient ainsi de convenir que l'assurance d'une gestion efficience des transactions multiétapes, par état complexe, ne peut s'envisager sans l'aide de "systèmes d'information hyper-performants ». Dans le cas ici étudié, nous proposons un modèle conceptuel à partir des préconisations de Laudon et Laudon (2001) pour la mise en place d'un Système d'Information de Gestion. L'objet est de lier le transport de marchandises dans une vision systémique de chaîne d'approvisionnement des points de vente urbains. Nous appliquerons ce modèle au cas du groupe Nouvelles Messageries de la Presse Parisienne (NMPP) dans le cadre du projet Logistique Urbaine Mutualisée Durable (LUMD), qui se trouve dans sa phase de développement après la définition des objectifs du projet et la mise en place d'un groupe de réflexion pluridisciplinaire en janvier 2009. Les principaux résultats présentés ici sont issus du travail demandé au Laboratoire d'Economie des Transports sur une analyse des marges de manœuvre tactiques et opérationnelles au sein du groupe NMPP pour une meilleure mutualisation de ses livraisons urbaines. 


\section{Revue de la littérature}

Les principes collaboratifs au niveau de la chaîne logistique se dessinent par étape, chaque étape correspondant à un degré d'engagement de plus en plus élevé. Quatre grandes étapes de collaboration peuvent être citées pour exprimer une mutualisation logistique, étapes qui concordent en même temps à des degrés de maturité (Baglin et al., 2009):

- la collaboration transactionnelle a comme objectif le traitement en commun des opérations administratives grâce à l'utilisation des Systèmes d'Information (S.I.). Elle apparaît comme un outil d'amélioration de l'efficacité par une gestion commune des échanges de documents administratifs ;

- la collaboration informationnelle concerne le partage d'informations telles que les prévisions de vente au consommateur final entre les points de vente, les distributeurs et les producteurs, etc. Le point crucial de cette collaboration est le respect de la confidentialité vis-à-vis de la concurrence ;

- la collaboration tactique et opérationnelle est liée à certains choix d'orientation à court et moyen terme qui peuvent toucher par exemple aux prévisions de production et de ventes, à la confection des plans de transport, aux approvisionnements et au niveau de qualité du service de distribution ;

- la collaboration stratégique, stade ultime, étend le champ décisionnel mis en commun à la chaîne de valeur dans son ensemble (R\&D, Marketing, processus de production et de distribution, etc.) et donc aux décisions commerciales et stratégiques des acteurs impliqués.

La mutualisation logistique peut avoir lieu dans les trois derniers stades de la collaboration, en commençant par la mise en commun d'informations. Si de nombreux travaux permettent de caractériser les principales pratiques collaboratives à l'intérieur d'une même chaîne d'approvisionnement (Barrat, 2004 ; Min et al., 2005 ; Simatupang et Sridharan, 2005 ; Khouider et al., 2006 ; Roy et al., 2006 ; Simonot et Roure, 2007 ; Livolsi, 2009), la mutualisation des flux de transport, qui se traduit par une mise en commun d'une partie de deux ou plusieurs chaînes logistiques, reste encore peu traité. Dans la littérature scientifique, ce terme est assez nouveau, et est souvent confondu avec la collaboration logistique (Simonot et Roure, 2007 ; Sboui, 2008). Le nombre d'études sur la mutualisation des flux de transport apparaît à ce jour limité (Nemoto, 2003 ; Simonot et Roure, 2007 ; Sboui, 2008 ; Tremeac et Raux, 2009). Etant un concept relativement nouveau, une définition standard n'a pas encore été énoncée. Nous proposons donc de définir la mutualisation des flux de transport comme "un type de collaboration inter-entreprise caractérisée par une mise en commun de ressources de deux ou plus acteurs (concurrents ou non) avec l'objectif d'une meilleure rationalisation des flux de transport. Par rationalisation des flux de transport, nous entendons tout moyen (économique, technologique, humain, organisationnel, etc.) susceptible d'améliorer la livraison d'un bien et service jusqu'à son point de consommation final. »

L'information étant à la base de toute mutualisation, il apparaît donc opportun de réfléchir au S.I. qui s'adapte au mieux aux questions que se pose la structure concernée par ce type de projet. A ce titre, il convient de rappeler avant tout en quoi consiste un S.I. La 
définition la plus communément utilisée dans ce cadre est proposée par Robert Reix (1995, p. 67) : "Un système d'information est un ensemble organisé de ressources : matériel, logiciel, personnel, données, procédures permettant d'acquérir, traiter, stocker, communiquer des informations (sous forme de données, textes, images, sons, etc.) dans des organisations». Dans cette veine, les bases organisationnelles des S.I. s'apprécient par la conjugaison de cinq éléments, à savoir la gestion, les technologies de l'information, l'organisation, les défis de l'entreprise et les solutions de l'entreprise (Laudon et Laudon, 2001). A terme, le but est d'atteindre une gouvernance des S.I. dont l'objet est «d'inciter les acteurs à repenser et modifier leurs pratiques de coordination et d'apprentissage pour contribuer activement à une dynamique d'innovation qui suppose confiance, partage d'information mais aussi confidentialité. » (El Amrani et al., 2008).

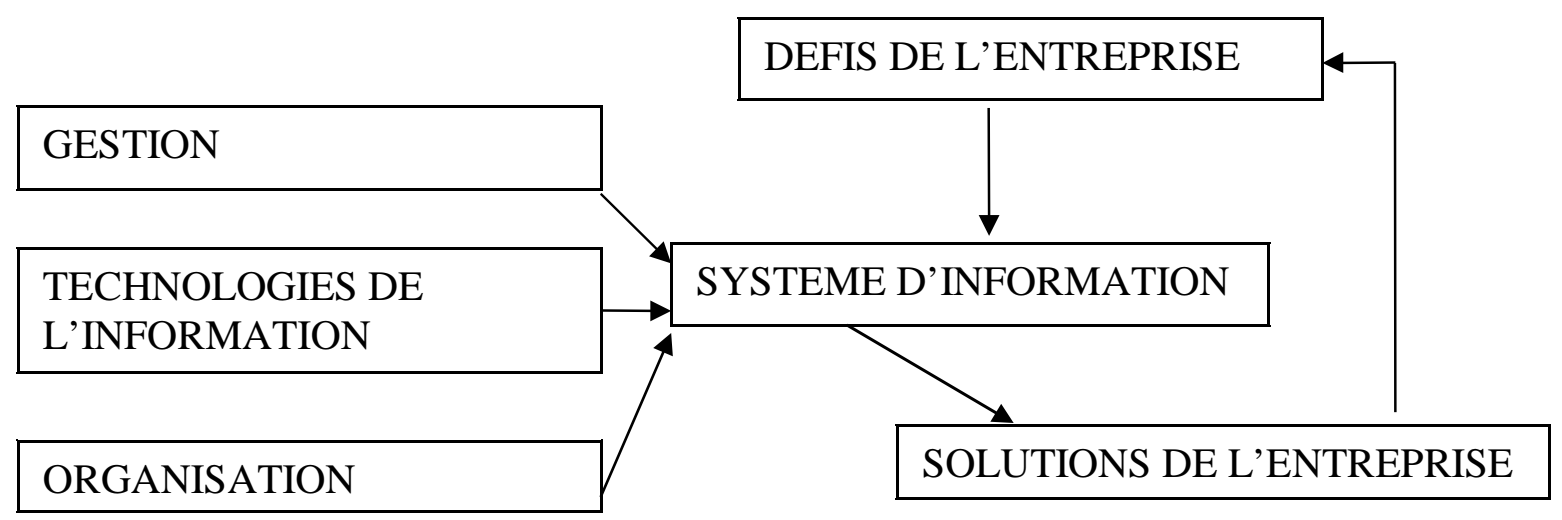

Figure 1 : Les bases organisationnelles d'un Système d'Information

(tiré de Laudon et Laudon, 2001)

En s'appuyant sur la représentation telle que montrée en Figure 1, il est possible d'identifier et de recenser les informations, méthodes et procédures utiles à la mise en place d'un S.I. à même de répondre aux préoccupations de l'acteur ou des acteurs définissant un projet de mutualisation de flux de transport. Dans cette perspective, le 'volet gestion' cherche à établir un panorama des travaux en rapport avec la mutualisation des flux de transport en marchandises. Le 'volet technologies de l'information' pose un regard sur les Technologies de l'Information du transport dans une vision supply chain. Le 'volet organisation' s'intéresse aux acteurs, tant internes comme externes, qui jouent un rôle prépondérant dans l'implantation et la pérennité du S.I. Selon nous, le volet 'défis de l'entreprise' constitue l'objet même du projet dans son ensemble. Par là, il faut entendre que ce volet effectue un état des lieux de la situation actuelle, la définition des problématiques que le projet doit affronter et la prise en compte des risques pour déterminer les objectifs du projet et les principales lignes d'action (par exemple, la mise en place du modèle économique ou les principales stratégies commerciales). Quant aux 'solutions de l'entreprise', c'est par une mise en place de Sustainability Balanced Scorecards (Hockerts et al., 2002 ; Naro et Noguera, 2005), que les acteurs du projet peuvent assurer une réussite de ce projet sur un plan économique, mais aussi social et environnemental.

(C) Revue Française de Gestion Industrielle 


\section{Notre protocole de recherche}

Les éléments présentés dans la première partie de ce travail nous permettent d'énoncer que le cadre d'un système de transports mutualisés pour la distribution urbaine de marchandises peut s'apprécier à travers la mise en place d'un S.I. Dans cette section, nous présentons un modèle général basé sur ces éléments. C'est en nous fondant sur les préconisations de Laudon et Laudon (2001) que ce modèle est exposé.

\subsection{Le 'volet gestion' ou la recherche d'une mutualisation des flux de transport de marchandises}

Nous pouvons observer deux modes de fonctionnement pour la mutualisation des flux de transport. Tout d'abord, le plus souvent utilisé mais peu étudié est ce qu'on peut appeler la «collaboration plurilatérale», i.e. différents acteurs du transport de marchandises se mettent d'accord pour mutualiser leurs ressources entre eux, avec ou sans accord de partenariat, mais en tout cas sans faire appel à une société intermédiaire pour la gestion de cette mutualisation. Ce système se base sur la confiance mais a des limites de fiabilité lorsque le nombre d'entreprises se mettant d'accord est grand, car elles ne sont pas cordonnées par un acteur dédié à la gestion de la collaboration. De fait, en soi, la mutualisation des flux de transport entre deux chargeurs ou transporteurs est une pratique très répandue mais n'a lieu que pour des situations particulières et n'a souvent pas vocation de pérennité (Tremeac et Raux, 2009). Néanmoins, plusieurs acteurs ont développé des stratégies à long terme fondées sur une mutualisation des infrastructures et des ressources avec des partenaires, concurrents ou non. Dans le cas d'entreprises non concurrentes, on peut observer le projet de mutualisation des ressources pour la distribution (y compris les flottes et le personnel chargé du transport) entre deux sociétés de l'industrie agroalimentaire qui ont en commun une partie de leur clientèle (Patier, 2004). Un autre projet qui porte sur une planification stratégique de la distribution est celui de deux entreprises concurrentes du secteur automobile qui ont réalisées un bâtiment commun de logistique pour mutualiser leur distribution dans un but de réduction des coûts liés à la distribution (Solard, 2008). Cette plate-forme est structurée en deux ailes (une pour chaque société) connectées par une partie commune pour la préparation des livraisons, réalisées par le même opérateur.

Un autre mode de fonctionnement s'est développé dans les dernières décennies : les " groupements de transporteurs », qui ont un statut juridique (normalement une association ou une société coopérative). Ils s'appuient sur un système centralisé d'échange d'information sur les marchandises à transporter en circuit fermé (Simonot et Roure, 2007). Ce système dérive du concept de "place de marché », une plate-forme d'échange virtuelle qui a comme but pal de fédérer les offres et demandes de clients et de vendeurs professionnels, dans un secteur ou pour des produits et/ou services spécifiques (Gensollen, 2001). Cette plate-forme technique est en général développée et géréepar un tiers, le 'maître de la place de marché', qui offre des mécanismes de transaction sécurisés permettant à des chargeurs de trouver des transporteurs proposant des services spécifiques de transport à des conditions tarifaires particulièrement intéressantes grâce à des systèmes d'enchères ou d'appels d'offres. 


\subsection{La part centrale des 'Technologies de l'Information et de la Communication' dans le transport}

Sur le plan de la gestion des transports, deux types de modules de T.I.C. sont déterminants : (1) les modules classiques de gestion et d'optimisation internes liés à l'activité de transport; (2) les modules qui permettent au transport de s'insérer dans les chaînes logistiques.

Les modules classiques de gestion et d'optimisation internes liés à l'activité de transport

Les décisions dans la planification tactique du transport de marchandises ont un impact direct sur la qualité du service mais aussi sur ses coûts (Crainic et Laporte, 1997). Ces deux facteurs sont liés, et il est important de les considérer dans la définition de la configuration du réseau de transport. En ce sens, le réseau de transport doit s'adapter au mieux aux contraintes économiques, géographiques, organisationnelles ou de qualité (Crainic, 2000 ; Wieberneit, 2008). Plus précisément, les questions principales qui se posent au sein des décisions tactiques sont liées (1) aux politiques d'approvisionnement et de stockage des entrepôts ; (2) à la configuration des tournées et la cadence du service ; (3) aux véhicules utilisés et (4) à l'affectation du personnel aux différents véhicules et tournées.

Dans la distribution de marchandises, le management des stocks et leurs politiques d'approvisionnement des entreprises de distribution ont des marges d'amélioration bien supérieures au transport, surtout dans les cas où ce dernier est sous-traité à des prestataires. Selon la taille de l'entreprise, les poids et volumes stockés, les contraintes spécifiques à la marchandise et d'autres facteurs, les entreprises de distribution peuvent faire appel à des logiciels de gestion des stocks et de planification des opérations d'entreposage. Ces logiciels utilisent des méthodes d'optimisation qui ont fait l'objet de nombreuses études (Goetschalckx et al., 2002 ; Dullaert et al., 2007). Or, il ne faut pas oublier les coûts de transport car ils constituent une composante importante de l'ensemble des coûts de la chaîne d'approvisionnement (d'après Toth et Vigo (2002), de 15 à $20 \%$ du coût final d'un produit). Les logiciels pour l'aide à la planification tactique et opérationnelle du transport de marchandises dérivent des méthodes de la recherche opérationnelle pour la résolution de problèmes d'optimisation des tournées des véhicules et des cadences du personnel (Crainic et Laporte, 1997 ; Leung, 2004). Ces problèmes ont connu des développements significatifs dans les dernières décennies et au jour d'aujourd'hui le nombre d'études scientifiques sont nombreuses (Toth et Vigo, 2002 ; Leung, 2004 ; Barnhart et Laporte, 2007 ; Golden et al., 2008). A partir de ces études, de nombreux logiciels d'optimisation ont été créés, et sont utilisés par la plupart des opérateurs de transport en groupage (Hall et Partyka, 2008).

Par contre, l'optimisation à court terme n'est pas pratiquée par toutes les entreprises de distribution. Si bien que si dans la gestion des stocks, une démarche de suivi en temps réel est souvent appliquée; dans les transports, l'optimisation en ligne des tournées n'est pas une pratique très diffusée. Néanmoins, avec les nouvelles technologies et notamment les systèmes de traçabilité véhicule, des outils d'optimisation des parcours souples et rapides ont été conçus (Hall et Partyka, 2008). 
Les modules qui permettent au transport de s'insérer dans les chaînes logistiques

Il apparaît donc comme incontournable, voire incontestable que les T.I.C. liées à une "gestion classique » d'une activité de transport jouent un rôle clé dans une meilleure lisibilité d'une chaîne d'approvisionnement. Même si, à ce jour, les questions de coûts et d'interopérabilité demeurent une préoccupation attachée à toute T.I.C., leur prise en compte constitue un pré-requis à tout échange. Au vu de l'élargissement des tâches du transporteur, et outre les modules classiques de gestion d'une activité de transport, une attention soutenue doit également être portée sur les technologies associées aux modules de S.I. qui permettent au transport de s'insérer dans les supply chains. Dans ce cadre, Fabbe-Costes (2007) en identifie trois catégories : les systèmes d'échange de documents, les systèmes de communication et les systèmes de traçabilité (Tableau 1). Tout d'abord, les systèmes d'échange de documents ont pour rôle d'assurer l'échange d'informations logistiques entre acteurs, mais surtout de "mémoriser» les différentes tractations entreprises. Ensuite, les systèmes de communication assurent le guidage des flux de marchandises entre les acteurs. Enfin, les systèmes de traçabilité visent principalement à situer précisément le « déplacement de la marchandise ».

\begin{tabular}{|l|l|}
\hline Systèmes d'Information & Outils / Technologies \\
\hline $\begin{array}{c}\text { Systèmes d'échange de documents } \\
\Rightarrow \text { Ordre de transport, avis de livraison, } \\
\text { bon de livraison, récépissé transport, } \\
\text { connaissement, liste de colisage.... }\end{array}$ & $\begin{array}{l}\text { Fax automatique ; Minitel ; EDI ; Internet (e- } \\
\text { mail, web-EDI, communication on-line }\end{array}$ \\
\hline Systèmes de communication & $\begin{array}{l}\text { Radio embarquée ; Terminaux embarqués ; } \\
\text { portable ; Téléphone ; Téléphone mobile ; } \\
\text { Internet (e-mail, communication on-line) ; } \\
\text { Terminaux portables multifonctions }\end{array}$ \\
\hline Systèmes de traçabilité & $\begin{array}{l}\text { Identification/codification ; Marquage ; Lecture } \\
\text { électronique ; Balises ; Systèmes vocaux ; } \\
\text { Enregistreurs ; Mémorisation }\end{array}$ \\
\hline
\end{tabular}

Tableau 1 : S.I. et T.I.C. du transport pour une insertion dans les chaînes d'approvisionnement (adapté de Fabbe-Costes, 2007)

\subsection{Le 'volet organisation' dans la (re)connaissance de l'acteur}

Or, à quelque niveau de la strate organisationnelle, l'acteur a un rôle névralgique dans le processus de réalisation du S.I. Qu'il appartienne à l'entreprise ou qu'il entreprenne des relations avec d'autres acteurs avec d'autres entreprises, il convient pour chaque acteur d'appréhender avant même l'implantation du S.I. ses composants, ceci afin d'en assurer plus aisément son adoption (Meissonier et Houzé, 2008). En ce sens, plusieurs éléments sont à considérer pour assurer l'acceptation d'une technologie de l'information (Davis et al., 1989). Parmi les plus significatifs, la communication en tant que facteur d'adhésion des utilisateurs au changement et de partage de l'information, la formation, la gestion des compétences mais également une implication des utilisateurs sont à considérer comme des facteurs véhiculaires dans la mise en place de toutes technologies de l'information, y compris celles en lien avec la logistique (Worou, 2008). 


\subsection{Les 'défis de l'entreprise' dans une prise en compte des risques inhérents à la mise en ouvre d'un S.I.}

Considérant les outils et technologies nombreux en transport (EDI, RFID, TMS, etc.) et de leurs niveaux d'utilisation (opérations, gestion des processus, interface entre acteurs/étapes, aide à la décision), des choix doivent être pris pour déployer un S.I. de transport. Par là, il faut entendre que toute organisation, toute chaîne d'approvisionnement n'a ni pour vertu, ni pour intérêt de solliciter toutes les technologies à sa disposition. Il faut donc poser la/les question(s) qui répondent aux attentes en termes de transport (et de logistique !) qu'elles -organisations et chaîne(s) d'approvisionnement- se sont fixées.

En conséquence de quoi, tout projet de mise en place d'un (nouveau) S.I. de transport requiert une analyse fine des risques encourus. A ce titre, Seiersen (2006) signalent six types de risques à gérer dans la mise en œuvre d'un S.I. :

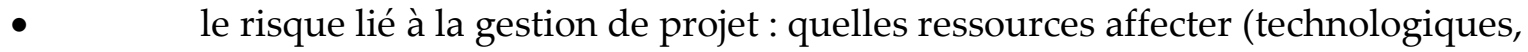
financières, humaines, partenariales) ?;

- $\quad$ le risque lié à l'organisation et à sa pérennité : quel est l'ordonnancement, le plan du projet? ;

- $\quad$ le risque lié aux technologies mises en œuvre car rares, voire inexistantes sont les technologies achetées qui répondent dès l'achat aux tâches attendues : des problèmes de fonctionnalité, de robustesse, de compatibilité sont 'monnaie courante'.

- $\quad$ le risque lié aux politiques, aux processus et aux pratiques actuelles. Tout nouvel S.I. entraîne un changement dans la façon de faire, de penser. Une réflexion sociale est inévitable avant, pendant et après tout projet de mise en place d'un S.I.

- le risque lié à l'impact des systèmes sur les opérations (opérations humaines, techniques).

- $\quad$ le risque lié à une plus grande dépendance par rapport aux systèmes en cas de défaillance.

\subsection{La 'solution recherchée' dans une réflexion fondée sur le concept de Développement Durable}

La notion de Développement Durable (DD) devient un discours de référence pour les entreprises. Plusieurs normes et référentiels sont actuellement proposés et utilisés pour répondre aux trois volets économique/social-sociétal/environnemental que constituent ce dernier. Dans la réflexion menée ici, l'intérêt se porte sur la définition et la pratique d'indicateurs sociaux, environnementaux et économiques (Depoers et al., 2003) et qui répondent ici, pour le projet LUMD, à trois points essentiels, à savoir le transport des marchandises, les espaces logistiques et les services et qualité de vie. En ce sens, l'objectif est alors de mettre alors en place ce qu'on appelle des Sustainabilily Balanced Scorecards (Hockerts et al., 2002 ; Naro et Noguera, 2005).

En résumé, les éléments présentés dans ce paragraphe répondent à une problématique d'identification des variables nécessaires au développement d'un S.I. apte à assurer une correcte mutualisation des flux de transport (Cf. Figure 2). 


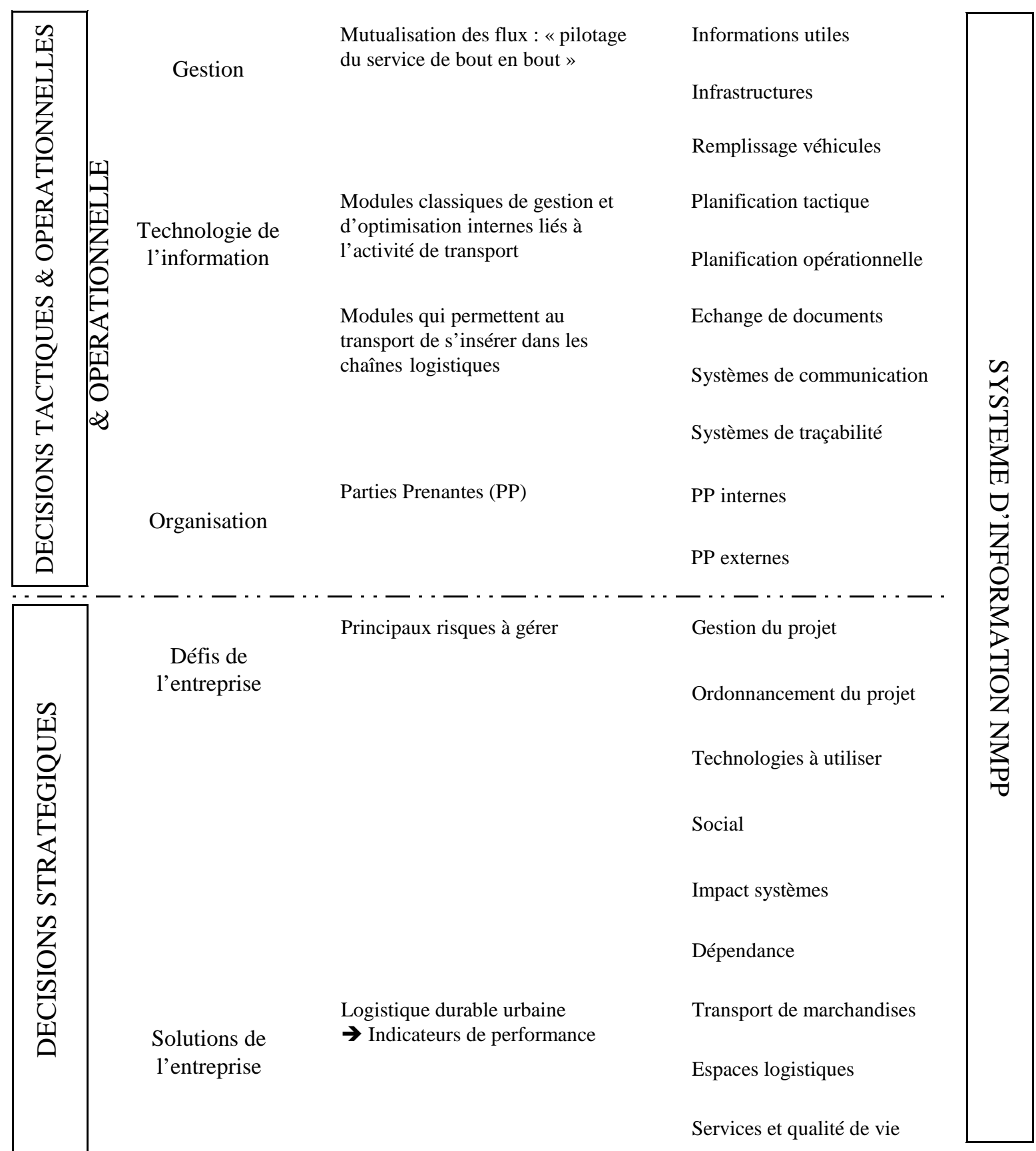

Figure 2 : Mise en place d'un système de mutualisation de transport pour le groupe NMPP

\section{L'Etude de terrain : les marges de manœuvre du groupe NMPP}

Le cas d'étude proposé s'intéresse au groupe NMPP. Les Nouvelles Messageries de la Presse Parisienne est le nom d'une société commerciale de type SARL structurée à hauteur de 51\% par cinq coopératives de presse (Express-Roularta, Prisma Presse, Marie-Claire, Le Nouvel Observateur, DI Group) qui arrêtent les barèmes de vote selon le principe 1 éditeur $=1$ voix ; et à hauteur de $49 \%$ du groupe Lagardère qui assure la Direction Générale en tant qu'opérateur. Son métier est centré sur les activités de distribution de la presse écrite en 
France, i.e. l'accueil, la répartition, la distribution, l'aide à la vente et le rendre compte de la presse quotidienne nationale, les magazines et de l'assimilé presse tels que les collectionnables, les figurines et autres gadgets. Le secteur de la messagerie de presse a un statut particulier. En France, seules deux sociétés ont la dénomination de messagers de presse : les NMPP, qui représentent $80 \%$ des volumes transportés (dont la quasi-totalité de la presse écrite quotidienne, à l'exception d'une partie peu significative de quotidiens régionaux), et les Messageries Lyonnaises de Presse (MLP), qui ne distribue pas de quotidiens et dont les volumes transportés sont d'environ $20 \%$. A noter que la presse gratuite n'est pas distribuée par les messageries de presse mais utilise des canaux spécifiques.

\begin{tabular}{|c|c|}
\hline \multicolumn{2}{|c|}{ Quelques chiffres-clés } \\
\hline CA 2008 & $2560 \mathrm{M} €$ \\
\hline CA 2007 & $2677 \mathrm{M} €$ \\
\hline \multicolumn{2}{|c|}{3000 salariés en CDI à fin 2008} \\
\hline \multicolumn{2}{|c|}{2.5 millions de clients par jour } \\
\hline \multicolumn{2}{|c|}{ Tonnages transportés par type de routage: } \\
\hline \multicolumn{2}{|c|}{ - $\quad 77 \%$ par route (404 000 tonnes) } \\
\hline \multicolumn{2}{|c|}{ - $\quad$ 19\% par ferré (100 000 tonnes) } \\
\hline \multicolumn{2}{|c|}{ - $\quad 2.5 \%$ par avion (13 000 tonnes) } \\
\hline \multicolumn{2}{|c|}{ - $\quad 1.5 \%$ par mer (8 000 tonnes) } \\
\hline \multicolumn{2}{|c|}{ Répartition des ventes fin 2008} \\
\hline \multicolumn{2}{|c|}{$\begin{array}{ll}\text { - } & \text { Ventes globales : } 1235 \text { Millions d'Exemplaires (2560 Millions } \\
& \text { d'euros) ; taux moyen d'invendus : } 41.3 \% \text { ( } 49.9 \% \text { en valeur) } \\
\text { - } & \text { Ventes dans les DOM }=1.1 \% \text { des ventes globales } \\
\text { - } & \text { Ventes export (100 pays) }=8.9 \% \text { des ventes globales }\end{array}$} \\
\hline
\end{tabular}

Tableau 2 : Chiffres clés du groupe NMPP

En 1994, face à une constante diminution des ventes de la presse écrite, le groupe NMPP a mis en place plusieurs stratégies à court terme afin de moderniser ses actifs. Les actions les plus importantes sont:

- la refonte du réseau de distribution et de la gestion des invendus (1994-1997), qui a eu comme effet une économie de plus de $120 \mathrm{M} €$ /an, au bénéfice des éditeurs $(80 \%)$ et des points de vente $(20 \%)$;

- le plan de réforme industriel et commercial (2002-2003) qui a permis de réaliser une économie récurrente d'environ $60 \mathrm{M} €$ /an au seul bénéfice des éditeurs, notamment par la refonte du réseau à Paris ;

- le nouveau plan stratégique de développement (2007), basé notamment sur l'amplification du réseau de points de vente et sur une refonte industrielle ;

- les stratégies de mutualisation (2007, 2009 et suite), qui ont permis la mise en place d'un groupe mixte de travail pour mettre en place une plate-forme logicielle dans le cadre du projet LUMD. 
Dans cette perspective, l'implication du groupe NMPP au projet LUMD a pour but (1) d'optimiser les "parties fixes " (surfaces plateformes/dépôts) non utilisées à ce jour et (2) d'enrichir la logistique pour y ajouter d'autres produits, ainsi que d'autres tournées. Pour le premier point, l'objectif est de remplir au maximum les camions, ce qui n'est pas le cas actuellement tous les jours. Sur le deuxième point, une filiale -Ocyto- a été créée en 2007. Cette filiale est un prestataire logistique qui, en tant que structure commerciale " indépendante », a pour but d'établir des contrats avec des entreprises autres que celles en lien avec la presse (démarche commerciale différente).

\subsection{Les résultats de l'étude qualitative menée sur les 5 premiers mois de 2009}

Nous présentons les éléments décisionnels tactiques et opérationnels du groupe NMPP dans les réflexions sur le projet LUMD.

\section{Le volet gestion : la mutualisation des flux}

\section{La structure de distribution}

Le système logistique du groupe NMPP se divise en trois parties: la distribution « amont « qui va jusqu'aux 172 dépositaires de presse de NMPP, la distribution « aval « qui va jusqu'aux 30000 points de vente, et un flux de « retour » des invendus (Cf. Figure 4) :

- la distribution «amont» combine plusieurs types de distribution, qui s'appuie sur les infrastructures suivantes : deux centres nationaux de groupage et traitement de la presse écrite (un pour les quotidiens et un autre pour les publications), des centres régionaux de quotidiens et des plates-formes régionales, auxquels se rajoutent les 172 dépôts de presse et un centre de diversification pour le groupage et la distribution aux plates-formes régionales de produits hors presse (gadgets) distribués par les mêmes canaux aux points de vente. La distribution «amont» présente une grande flexibilité et de nombreuses combinaisons pour la distribution des produits. Ainsi, le groupe NMPP a établi des partenariats avec deux sociétés de transport pour la distribution «amont». L'organisation des transports est à la charge des NMPP, qui passent chaque jour les commandes pour le transport, en privilégiant le transport en camion complet.

- la distribution « aval » est effectuée avec des véhicules légers (ex. : fourgons) détenus par plusieurs petites entreprises de transport. Le système de tournées est relativement rigide. Il dérive des fortes contraintes du métier (exemple: les quotidiens sont en "flux tendus », les autres produits type mensuels sont en "flux programmés "; livraison aux points de vente avant $6 \mathrm{~h}$ du matin; problèmes de sécurité par des vols avant l'ouverture des points de vente). Compte-tenu de l'ensemble de ces contraintes (multiples transporteurs, contraintes métier), les tournées sont peu flexibles et rendent difficilement maîtrisables les taux de remplissage dans une planification à moyen terme. En Ile de France, la distribution «aval » se fait en deux tournées par point de vente : la première est utilisée pour la livraison des publications et la deuxième pour les quotidiens et éventuellement les produits non livrés dans la première tournée, dans les cas où les volumes des 
produits en flux programmé seraient supérieurs aux capacités des véhicules.

- Enfin, un flux « retour» des invendus est traité directement par le groupe NMPP, soit sous forme de recyclage vieux papiers (environ 90\% des invendus), soit en retour chez les éditeurs ou en remise sur le circuit commercial à l'exportation, par exemple (environ $10 \%$ des invendus).

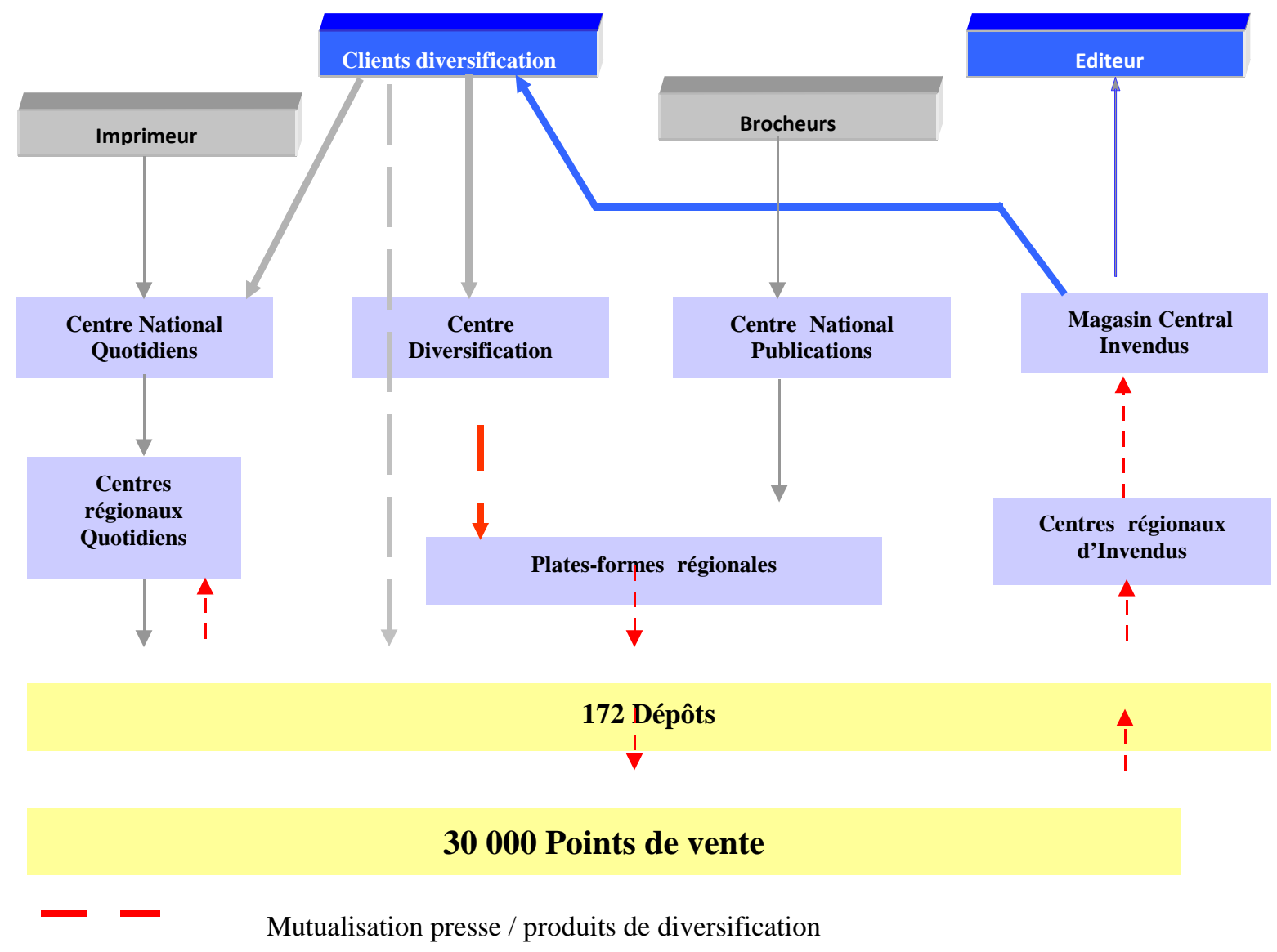

Figure 3 : Les principales possibilités de mutualisation des flux de transport dans les schémas logistiques du groupe NMPP (source NMPP, 2009)

Or, un des objectifs recherchés à travers le projet LUMD est de réfléchir à la diminution des ruptures de charge. Une redéfinition du réseau de distribution «amont » serait «d'éliminer» certaines plates-formes qui n'effectuent que de simples transferts de journaux et induisent des coûts élevés. Ce faisant, pour l'instant, la mutualisation recherchée par le groupe NMPP est de prendre une plus grande place sur son circuit « aval ». Mais, à ce niveau, le groupe NMPP est confronté à deux problèmes :

- le premier est le respect des contraintes propres de la presse écrite, qui exclue certains produits, soit par incompatibilité du type de marchandises et de conditionnement (alimentaire frais ou congelé, certains liquides et d'autres marchandises dangereuses ou très fragiles), soit par les contraintes de temps de livraison de la presse. Dans ce cas, le groupe étudie la possibilité de prendre des commandes de transport qui ont des délais de livraison flexibles.

- Nous observons aussi un problème de sécurisation. En effet, au moment de la remise 
de la presse auprès des points de vente, beaucoup ne sont pas ouverts et plusieurs distributeurs peuvent avoir accès au même sas. Malheureusement, si le groupe NMPP est leader dans la diffusion de la presse, d'autres entreprises se partagent ce secteur d'activité (par exemple, les MLP). Chacun de ces prestataires a alors accès au même «sas » de dépôt et, de fait, aucune traçabilité si une « disparition » de produits ne peut être menée. La question de «sas dédié » à chaque prestataire constitue une réflexion prégnante pour des entreprises comme le groupe NMPP, par exemple, par le biais de «sas en propre NMPP» au-niveau des gares ....mais, ici se poserait la question du prélèvement par de multiples points de vente !.

En bref, le groupe NMPP réalise déjà des transports mutualisés en s'appuyant sur sa filiale Ocyto : les transports dérivent d'accords à moyen terme entre les deux parties (NMPP et son interlocuteur correspondant, pour chaque commande). Ces accords sont en général effectués dans le cadre de transports réguliers et à un horizon à moyen terme. Actuellement, deux sociétés collaborent avec les NMPP pour mutualiser leurs produits éditoriaux ayant comme destination les points de vente qui ont des points communs avec la presse écrite. Deux possibles développements sont prévus: d'un côté, une augmentation du nombre de clients d'Ocyto, livrant ou non les mêmes points de vente que ceux desservis actuellement par le réseau du groupe NMPP ; d'un autre côté, la mise en place de la plate-forme LUMD, pour attirer d'autres clients réalisant des transports réguliers ou ponctuelles. Le projet LUMD tiendra compte de trois types de flux de transport: les flux réguliers, les flux ponctuels non urgents, et enfin les urgences. Cette plate-forme devrait permettre au groupe de diversifier son réseau de distribution et d'augmenter les taux de chargement des véhicules, principalement ceux du circuit aval (des dépôts de presse aux points de vente).

\section{Le volet technologies}

\section{Le système de gestion des tournées et de design du réseau}

Si le recours à des systèmes par satellite s'avère prépondérant dans le cadre du transport industriel où les trajets sont variés et multiples, cette problématique n'a pas à ce jour de sens dans le cadre du groupe NMPP. En effet, la gestion des tournées auprès des diffuseurs est fixe et ne subit que rarement de variations (ex. : travaux routiers, événements sportifs). Dans le cadre de sa gestion des tournées, le groupe NMPP détient des logiciels internes adaptés à son métier: le logiciel Winroute, commercialisé par Routing International, intègre des techniques avancées en termes de planification et d'optimisation. En ce qui concerne les flux arrivant aux dépôts de presse, la gestion pour la presse hors quotidiens est planifiée à l'aide du logiciel interne OVT, basé sur des règles métier très précises. De même, sur un horizon à moyen terme et dans une diversification des produits, d'autres outils de planification sur des stratégies de distribution plus souples ne pourraient être envisagés qu'à la condition d'une augmentation fort conséquente de nouvelles activités du groupe (en termes de volumes, valeurs et points de livraison).

Un autre système de gestion des tournées pourrait trouver place dans cette catégorie. Il s'agit de l'impact de l'amélioration des technologies qui touche le véhicule en propre. 
Ainsi, la contrainte énergétique impose de réfléchir à des modes de consommation moindres. Un changement de motorisation est une solution envisageable. Or, le groupe NMPP ne détient aucun véhicule en propre et passe donc par une sous-traitance. Même si sa volonté est de diminuer le taux de pollution, son action est extrêmement faible à ce niveau. Alors que les livraisons par nuit correspondent à un trajet global équivalent à 1 tour $1 / 2$ de la Terre, difficile est de trouver de solutions. Ce faisant, et de manière plus générale, l'action du groupe NMPP est de tenter une diminution de l'usage du fret aérien (fort consommateur d'énergie et qui était une image de marque d'efficience par sa rapidité il y a peu) par l'usage du ferré (lignes de nuit sur Avignon et Montauban actuellement).

\section{Les systèmes de communication}

Les systèmes de communication vers les transporteurs sont extrêmement simples. Le transport s'effectuant en compte d'autrui, seul le téléphone portable est utilisé. En effet, la majorité des transporteurs sont des PME ayant des flottes de véhicules légers dont l'investissement en terminaux embarqués, par exemple, ne s'avère pas rentable. Entre les dépôts de presse et les autres plates-formes logistiques du groupe, il convient de noter la mise en place d'un système Wifi.

\section{Les systèmes d'échange de documents}

Les échanges de documents s'effectuent principalement avec des moyens traditionnels (fax) à l'aide du logiciel interne OVT. Cet outil gère quotidiennement le flux de marchandises de la presse qui arrive à chaque dépôt, et prépare les plans de transport. Actuellement OVT ne permet pas des échanges informatisés des données (EDI). Le flux retour des invendus reste pour l'instant sur une approche «manuelle».

Les opérations logistiques ont été particulièrement facilitées par l'introduction du système EDI dont l'optique est de favoriser les transactions commerciales entre acteurs d'une chaîne d'approvisionnement. Pour l'instant, le groupe NMPP ne compte qu'un système de communication interne entre centres de traitement et dépôts. Aucun EDI n'est disponible avec les acteurs externes. La mise en place d'un logiciel SAP est en cours dont le but est de suivre le référentiel des produits et assimilé produits issus de la presse. A terme, la volonté du groupe est d'utiliser ce progiciel SAP pour passer sur EDI.

\section{Les systèmes de traçabilité}

Parmi les préoccupations des entreprises, tous secteurs confondus, la question de la traçabilité est omniprésente. Sur ce type de produit, la question sur la santé de l'individu est faible, comme pour le secteur de l'alimentaire. Nonobstant, un système de traçabilité sur le système aval entre les dépôts de presse et les diffuseurs s'établit à travers un code à barre unidimensionnel. Sur un horizon à moyen terme, la question de la Rfid se pose sur des produits à valeur unitaire supérieure à 40 euros telles que les vidéos.

\section{Le volet organisation}

Les infrastructures du groupe NMPP s'apprécient via la détention de dépôts. Sur 172 dépôts sur le sol français, 60 appartiennent au groupe NMPP. Les 112 restants sont des 
dépositaires de presse indépendants qui sont propriétaires et partenaires -sous contrat de distribution- avec le groupe NMPP. Il convient de noter la spécificité du métier de la presse qui en fait un «métier fermé » et qui, en conséquence, limite fortement l'ouverture à la concurrence de nouveaux dépôts. Un autre élément crucial du mode de relation entre dépôt NMPP et dépôt indépendant est que l'engagement n'est pas fondé sur le volume. Par là, il faut noter que le «contrat partenarial » émis entre ces deux acteurs ne s'appuie pas sur une rémunération variable, en fonction de la saisonnalité et/ou de la quantité à livrer.

Parmi les pistes de recherche du groupe NMPP quant à la mutualisation de ses besoins, une réflexion voit le jour dans l'idée d'une utilisation de ces dépôts en propre aux horaires non utilisés dans le cadre du traitement de la presse qui sont du 20 heures à 8 heures du matin. La configuration actuelle n'est pas (ou du moins une étude au cas par cas de la surface de chaque dépôt est nécessaire) de réfléchir à un mode de gestion par entreposage. Toutefois, une approche selon une activité de cross-docking est tout à fait possible, surtout dans l'idée d'une extension à d'autres activités de produits gérées par la filiale Ocyto.

Un autre aspect, déjà mis en avant dans la description du système de distribution, est la sous-traitance des activités de transport. Le circuit amont étant plus flexible, des grands transporteurs réalisant des transports en camion complet peuvent être des collaborateurs habituels, sous forme de partenariat, et avec des plans de transport variables d'un jour à l'autre. Par contre, le système de distribution du circuit aval, composé d'un réseau de PME avec des petites flottes, et les contraintes de la distribution des quotidiens imposent des plans de transport peu souples et réorganisés tous les six mois. Des livraisons moins contraignantes, qui puissent compléter les espaces vides des véhicules du circuit aval, doivent cependant être mises en cuvre dans les possibles évolutions du projet de mutualisation.

\section{Les volets défis et solutions de l'entreprise}

Bien que n'étant pas du ressort du travail demandé au Laboratoire d'Economie des Transports, nous précisons quelques éléments clés aux décisions stratégiques.

Tout d'abord, les défis s'appréhendent selon six éléments : (1) la gestion du projet constitue l'objet même du projet LUMD dans son ensemble, elle est ici composée -comme préalablement souligné- d'acteurs d'horizons divers (groupe NMPP, universités, cabinets d'expertise, etc.), (2) l'ordonnancement du projet suit un programme d'environ une réunion mensuelle où les avancements des travaux sont appréciés, les quatre derniers éléments (technologies à utiliser, social, impacts systèmes et dépenses) sont en cours d'évaluation et suivront principalement la remise d'un document de synthèse sur l'état des lieux du groupe NMPP en septembre 2009.

Ensuite, à terme, l'objectif est de mesurer l'impact de ces actions. Sur ce volet, trois axes avaient été retenus à l'origine: (1) les activités de transport de marchandises, (2) les 
espaces logistiques et (3) les services et qualité de vie. La performance du projet LUMD sera évaluée par la mesure de paramètres sur les dimensions économique, environnementale et sociétale, dans des tests définis dans une phase avancée, prévue courant 2010. La prise en compte des effets sur la réduction du $\mathrm{CO}_{2}$ est jugée comme l'un des critères environnementaux les plus importants pour l'appréciation des choix logistiques opérés à l'aide de la plateforme.

\section{Conclusion}

Dans cet article, nous avons réalisé un état des lieux du système de distribution de la presse du groupe NMPP, ainsi qu'une analyse qualitative de la possible mise en place d'un projet de mutualisation des flux de transports basé sur un système d'information (S.I.). Si la durabilité peut s'apprécier à travers la notion de développement durable qui connaît un essor dans les discours actuels; il faut également y voir la notion de pérennité : pérennité qui est l'objet de toute entreprise. L'étude du cas du groupe NMPP est intéressante en ce sens. En effet, la décision d'entériner pour le groupe NMPP une démarche de Logistique Urbaine Mutualisée Durable (LUMD) constitue, pour ses dirigeants, ses salariés et ses partenaires, un besoin vital en vue d'une articulation des différents modes de fonctionnement et de régulations des demandes des clients finaux. De fait, le groupe NMPP est conduit à se doter de nouveaux instruments de régulation pour instaurer des échanges constants tout au long de «sa » journée de travail : la nuit, la presse ; le jour, les autres activités. A terme, l'amélioration des flux d'échange permettrait d'entériner des actions sur le transport des marchandises, les espaces logistiques urbains utilisés et les services et qualité de vie.

L'objectif de cet article était de regarder en quoi une correcte appréhension d'un système d'information joue un rôle primordial dans la mise en place d'un projet LUMD. Pour cela, à travers l'étude du groupe NMPP, nous avons tenté de rapprocher les axes de réflexion actuels du groupe NMPP pour permettre de répondre au mieux aux questionnements posés dans ce projet. Ce faisant, d'ores et déjà, il convient ici de noter que notre propos est exploratoire et descriptif compte-tenu de la relative "jeunesse » de la réflexion menée par le groupe NMPP dans cette direction, à savoir début de l'année 2009. Ce travail nécessitera donc une étude plus approfondie quant à la viabilité des actions suggérées et/ou entreprises. Ce faisant, et d'ores et déjà, la contrainte économique s'avère l'élément fondamental à une facilitation d'une mutualisation des flux de transport en milieu urbain, élément souligné par Paché (2009).

\section{Bibliographie}

Ambrosini C., Routhier J. L. (2004), Objectives, methods and results of surveys carried out in the field of urban freight transport: an international comparison, Transport Reviews, $\mathrm{n}^{\circ}$ Vol.4, n¹, pp. $57-77$.

Barnhart, C. et Laporte, G., eds., (2007), Transportation, Elsevier, North Holland.

(C) Revue Française de Gestion Industrielle 
Barrat, M. (2004), Understanding the meaning of collaboration in the supply chain, Supply Chain Management: An International Journal, Vol. 9, n 1, pp. 30-42.

Boudoin D. (2006), Guide de mise en cuvre d'espaces logistiques urbains, CRET-Log PREDIT.

Crainic, T. G. et Laporte, G. (1997), Planning models for freight transportation, European Journal of Operational Research, Vol. 97, pp. 409-438.

Crainic, T. G. (2000), Service network design in freight transportation, European Journal of Operational Research, Vol. 122, pp. 272-288.

Crainic, T. G. (2008), City Logistics, in Chen, Z. L. and Raghavan, S. (eds.), Tutorials in Operations Research 2008. State-of-the-Art Decision Making Tools in the InformationIntensive Age, INFORMS, e-book, pp. 181-212.

Dablanc, L. (1998), Transport de marchandises en ville. Une gestion publique entre police et services, Liaisons.

Davis, F.D., Bagozzi, R.P. et Warshaw, P.R. (1989), User acceptance of computer technology: a comparison of two theorical models, Management Science, Vol. 35, n 8, pp. 982-1003.

Delaître, L. (2008), Méthodologie pour optimiser le transport de marchandises en ville: application aux villes moyennes et dans le cadre de l'agglomération de La Rochelle. Monographie de Thèse. Ecole des Mines Paritech.

Depoers, F. Reynaud, E. et Schneider Maunoury, G. (2003), Comment mesurer la performance durable des entreprises? Proposition d'une grille d'indicateurs, Gestion 2000, mars-avril, pp. 13-29.

Dullaert, W., Bräysy, O., Goetschalckx, M. et Raa, B. (2007), Supply chain (re)design: Support for managerial and policy decisions, European Journal of Transport and Infrastructure Research, Vol. 7, n², pp. 73-92.

El Amrani, R., Clergeau, C. et Bidan, M. (2008), Le rôle du système d'information dans le pilotage d'un pôle de compétitivité, Colloque Association Internationale de Management Stratégique, Nice.

Fabbe-Costes, N. (1992), Les systèmes d'information et de communication au cœur de la stratégie des prestataires logistiques?, Selected Proceedings of The $6^{\text {th }}$ World Conference on Transport Research, Lyon, pp. 319-330.

Fabbe-Costes, N. (1994), Le processus logistique : support fécond d'une démarche de reengineering et lieu d'apprentissage organisationnel, $4^{\text {ème }}$ Rencontres MCX, Aix-en- 
Provence.

Fabbe-Costes, N. (1997), Pilotage logistique, Quel système d'information ?, in Aurifeille et al., Management Logistique : Une approche transversale, Ed. Litec, Paris, pp. 111-144.

Fabbe-Costes, N. (2000), Le rôle transformatif des SIC et des TIC sur les interfaces multiacteurs de la distribution et de la logistique, in Fabbe-Costes, N., Colin, J. et Paché, G. (Coord.), Faire de la recherche en logistique et distribution, Coll. FNEGE. Vuibert, pp. 171193.

Fabbe-Costes, N. (2007), Systèmes d'information logistique et transport, Techniques de l'Ingénieur.

Gensollen, M. (2001), Internet. Marché électronique ou réseaux commerciaux ? Revue économique, Vol. 52, n 1, pp. 137-161.

Goetschalckx, M., Vidal, C.J. et Dogan, K. (2002), Modelling and design of global logistics systems: A review of integrated strategic and tactical models and design algorithms, European Journal of Operational Research, Vol. 143, pp. 1-18.

Golden, B. L., Raghavan, S. et Wasil, E. A. (2008), Vehicle routing: Latest advances and challenges, Kluwer, Boston, USA.

Hall, R. et Partyka, J. (2008), On the road to mobility, OR/MS Today, Vol. 35, n 1, available online at: http://www.lionhrtpub.com/orms/orms-2-08/frvrss.html.

Hockerts, K., O'Rourke, A. et Zingales, F. (2002), Balanced scorecard and sustainability: state of art review, W.P. INSEAD 2002-65/CMER.

Khouider, S., Monteiro, M. et Portmann, M.-C. (2006), Gestion collaborative et distribuée des approvisionnements intégrant le transport, Conférence Internationale Francophone d'Automatique Cifa 2006.

Laudon K. et Laudon J. (2001), Les systèmes d'information de gestion. Organisation et réseaux stratégiques, Pearson Education France, Ed. Village Mondial Paris.

Leung, J. T. (2004), Handbook of scheduling: algorithms, models and performance analysis, CRC Press, Florida, USA.

Livolsi, L. et Fabbe-Costes, N. (2004), La centralité des systèmes d'information (S.I .) dans la fonction logistique, Revue Française de Gestion Industrielle, Vol. 23, n 4, pp. 27-44.

Livolsi, L. (2009), Le Supply Chain Management : Synthèse et propositions, Actes de la XVIII ${ }^{\text {ème }}$ Conférence Internationale Management Stratégique AIMS, Grenoble, 2-5 juin.

(C) Revue Française de Gestion Industrielle 
Meissonier, E. et Houzé, E. (2008), La non gestion des conflits dans le cadre de l'implantation des technologies de l'information: un cas de résolution autonome, Conférence Association francophone de Gestion des Ressources Humaines, Dakar, Sénégal.

Min, S., Roath, A. S., Daugherty, P. J., Genchev, S. E., Chen, H., Arndt, A. D. et Richey, R. G. (2005), Supply Chain Collaboration: What's Happening, International Journal of Logistics Management, Vol. 16, n², pp. 237-256.

Naro, G. et Noguera, F. (2005), Responsabilité sociale de l'entreprise et développement durable: quelle intégration possible dans le système de pilotage interne de l'entreprise ? de l'approche socio-économique aux "sustainable balanced scorecards ", $3^{\text {ème }}$ Congrès de l'Aderse, 18-19 octobre, Lyon.

Nemoto, T. (2003), An Experimental Study on Cooperative Parcel Pick-up System Using the Internet in the central business district in Tokyo. In Taniguchi, E. and Thomson, R. G. (eds.), Logistics systems for sustainable cities, Elsevier, Amsterdam, pp. 309-320.

Paché, G. (2009), Quels impacts de la crise sur la logistique ?, Revue Française de Gestion, Vol. $35, \mathrm{n}^{\circ} 193$, pp. 51-57.

Patier, D. (2002), La logistique dans la ville. Celse.

Patier, D. (2004), La place du transport de marchandises en compte propre, rapport DRAST.

Patier, D., Dufour, J. G. et Routhier, J.L. (2007), Du transport de marchandises en ville à la logistique urbaine, Techniques de l'Ingénieur.

Patier, D. et Routhier, J.-L. (2009a), La logistique urbaine, acquis et perspectives, Les Cahiers Scientifiques du Transport, $\mathrm{n}^{\circ}$ 55, pp. 5-10.

Patier, D. et Routhier, J.L. (2009b), Une méthode d'enquête du transport de marchandises en ville pour un diagnostic en politiques urbaines, Les Cahiers Scientifiques $d u$ Transport, $\mathrm{n}^{\circ}$ 55, pp. 11-38.

Reix, R. (1995), Systèmes d'information et management des organisations, Vuibert.

Rosini, R. (2005), Progetto City Ports - Logistica urbana a Bologna: elementi per un progetto, Quaderni del servizio Pianificazione dei Trasporti e Logistica, Regione Emilia Romagna, Bologna, Italy.

Routhier, J.L. (2002), Du transport de marchandises en ville à la logistique urbaine, Synthèses et Recherches. 2001 plus, n 59, 67 p., DRAST.

Roy J., Landry S. et Beaulieu M. (2006), Collaborer dans la chaîne logistique : État des lieux. Cahier de recherche, $\mathrm{n}^{\circ}$ 06-01, HEC, Montréal. 
Sboui, S. (2008), La logistique mutualisée durable, Logistique et collaboration inter-entreprises: enjeux, méthodes et outils, Journée DRAST du 21 mai 2008.

Seiersen, N. (2006), Systèmes d'information logistique, Techniques de l'ingénieur.

Simatupang, T.M. et Sridharan, R. (2005), An integrative framework for supply chain collaboration, International Journal of Logistics Management, Vol. 16, n² 2, pp. 257-274.

Simonot, P.-Y. et Roure, J. (2007), Logistique collaborative. Une question d'avenir, Economica.

Spinedi, M., ed. (2008) Logistica urbana: dagli aspetti teorici alle applicazioni, Regione Emilia Romagna - City Logistics Expo, Bologna, Italie.

Taniguchi, E., Thomson, R. G., Yamada, T. et Van Duin, R. (2001), City Logistics - Network modelling and Intelligent Transport Systems, Elsevier, Amsterdam, The Netherlands.

Taniguchi, E. et Thomson, R. G., (2008), Innovations in City Logistics, Nova Science Publishers, New York, USA.

Toth P. et Vigo D., (2002), The vehicle routing problem, SIAM monographs on applied mathematics, Philadelphia, USA.

Tremeac, Y. et Raux, C., coord. (2009), FIDES : Flexibilité et Impacts de la Demande de transport des différents secteurs Economiques, et simulation de Scénarios d'évolution. Rapport Final. Contrat PREDIT.

Wieberneit, N. (2008), Service network design for freight transportation: a review, OR Spectrum, Vol. 30, pp. 77-112.

Worou, R.D. (2008), Impact des pratiques des ressources humaines sur l'acceptation de l'ERP dans les entreprises en Afrique : cas de deux entreprises en Afrique de l'Ouest, Association francophone de Gestion des Ressources Humaines, Dakar, Sénégal.

\section{Revues professionnelles :}

Baglin, G., Betbeze, P. et Lembert, T. (2009), La supplu chain collaborative : pratiques et perspectives. Le journal de la logistique, n. 63, pp. 26-32.

Solard, G. (2008), La logistique collaborative : comment ça marche, Stratégie Logistique, $\mathrm{n}^{\circ}$ 109 , pp. 27-41.

\section{Références internes :}

NMPP (2008). Rapport d'activités 2008. Nouvelles Messageries de la Presse Parisienne, Paris.

(C) Revue Française de Gestion Industrielle 